\title{
ОСНОВНЫЕ НАПРАВЛЕНИЯ ВОССТАНОВИТЕЛЬНОЙ ТЕРАПИИ В ПОСТ-КОВИДНОМ ПЕРИОДЕ
}

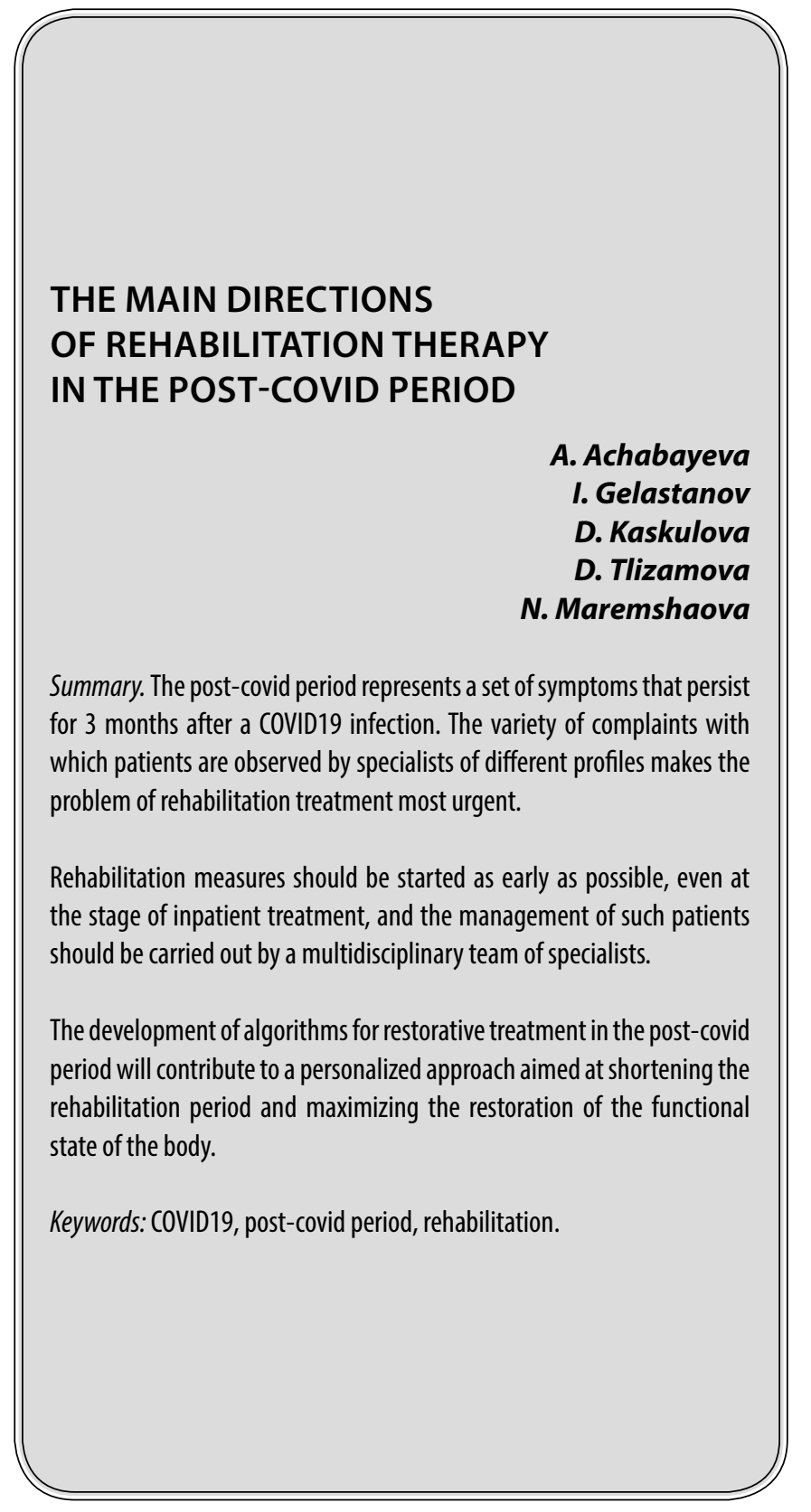

\section{Введение}

$\Pi$ ост-ковидный период представляет собой клиническое состояние, развитие которого наблюдается спустя несколько недель после перенесенной острой инфекции COVID19. При анализе результатов лечения пациентов с новой инфекцией было отмечено, что угрозу представляет не только острый период, но и вре-
Ачабаева Айшат Борисовна

Старший преподаватель, ФГБОУВО «КабардиноБалкарский государственный университет им. Х.М. Бербекова» Aisha.aca@yandex.ru Гелястанов Ибрагим Хизирович ФГБОУ ВО «Кабардино-Балкарский государственный университет им. Х.М. Бербекова»

Каскулова Диана Зауровна

ФГБОУ ВО «Кабардино-Балкарский государственный университет им. Х.М. Бербекова»

тлизамова Диана Аслановна

ФГБОУ ВО «Кабардино-Балкарский государственный университет им. Х.М. Бербекова»

Маремшаова Начна Арсеновна

ФГБОУ ВО «Кабардино-Балкарский государственный университет им. Х.М. Бербекова»

Аннотация. Пост-ковидный период представляет совокупность симптомов, сохраняющихся на протяжении 3-х месяцев после перенесенной инфекции COVID19. Многообразие жалоб, с которыми пациенты наблюдаются у специалистов разных профилей, делают проблему восстановительного лечения наиболее актуальной.

Реабилитационные мероприятия должны быть начаты как можно раньше, еще на этапе стационарного лечения, а ведение таких больных должно осуществляться мультидисциплинарной бригадой специалистов.

Разработка алгоритмов восстановительного лечения в пост-ковидном периоде будет способствовать персонифицированному подходу, направленному на сокращение сроков реабилитации и максимальному восстановлению функционального состояния организма.

Ключевые слова: COVID19, пост-ковидный период, реабилитация.

мя после него, даже у лиц, перенесших инфекцию в легкой форме.

Greenhalgh Trisha, et al. (2020) одними из первых предложили термин «long-covid», подразумевающий совокупность симптомов после перенесенного заболевания, которые сохраняются на протяжении более трёх недель с момента появления первых симптомов - «пост-о- 
стрый (вероятно имеется ввиду - подострый) COVID-19» и более 12 недель — «хронический COVID-19» [1].

Некоторые исследователи полагают, что постковидный период тяжелее протекает среди пациентов, имеющих сопутствующую патологию (ожирение, сахарный диабет, гипертоническая болезнь и т.д.) и определяется степенью поражения отдельных органов и систем в остром периоде.

Среди наиболее часто диагностированных жалоб, наблюдаемых специалистами в амбулаторном звене, являются: нарушение функции кардиореспираторной системы (одышка, тахикардия, утомляемость, головные боли) и желудочно-кишечного тракта.

Большинство авторов сходятся во мнении, что проявления пост-ковидного синдрома определяются системностью характерных поражений (изменения в одной системе ассоциированы с нарушением функциональной активности другой).

Данные, изложенные в разработанных специалистами здравоохранения Российской Федерации Методических рекомендациях (2020 г.) свидетельствуют о необходимости осуществления медицинской реабилитации пациентам, перенесших COVID-19 на всех его этапах: стационарном и амбулаторно-поликлиническом [2]. Кроме того, своевременность их применения будет вносить значимый вклад в выздоровление пациентов без развития тяжелых осложнений.

\section{Цель работы}

Анализ литературных данных, посвященный вопросам восстановительной терапии в пост-ковидном периоде.

Восстановительное лечение на первом этапе (в терапевтическом отделении стационара) должно быть направлено на раннее восстановление функционирования пациента. Важное значение уделяется выполнению дыхательной гимнастики, направленной на усиление эвакуации мокроты при ее наличии. В случае диагностирования у пациента нарушения дренажной функции бронхов может быть использован метод постурального дренажа [3].

При проведении дыхательной гимнастики со специалистом пациенту должен быть предоставлен кислород по требованию (баллоны с кислородом или устройства, продуцирующие кислород). Сроки начала выполнения дыхательных упражнений, назначаемых врачом, определяются тяжестью состояния пациента: легкое течение позволяет рекомендовать их спустя 1 неделю после начала заболевания; при среднетяжелом-на 8-й день госпитализации в случае положительной динамики клинической картины и данных визуализационных методов исследования (компьютерной томографии, рентгенологического исследования и т.д.) [4].

Еще одним мероприятием восстановительного лечения на данном этапе является применение последовательного изменения положения тела пациента относительно горизонтальной плоскости. Оно должно соответствовать требованиям, изложенным в Клинических рекомендациях Союза Реабилитологов России по вертикализации [5].

Поддержание нутритивного статуса на целевом уровне может быть обеспечено энтеральным или парентеральным питанием [6].

В течение всего времени пребывания в стационаре пациент должен находиться под наблюдением психотерапевта с целью проведения нейропсихологической реабилитации для минимизации риска развития тревожно-фобических расстройств [7].

На 12-14 дни нахождения в стационаре при отсутствии осложнений и диагностируемой положительной динамики с целью улучшения дренажной функции бронхов может быть применимо физиолечение-дозированная аэроионотерапия [8].

Реабилитация на амбулаторном этапе после перенесенного COVID-19 должна начинаться, по возможности, как можно раньше и включать следующие аспекты [9]:

- восстановление/сохранение функционального статуса,

- контроль за течением хронических неинфекционных заболеваний,

- поддержание мобильности и сохранение ментального здоровья,

- вакцинопрофилактику острых инфекционных респираторных заболеваний.

При составлении плана восстановительного лечения терапевтами первичного звена должное внимание должно уделяться выполнению дыхательной гимнастики в режиме нарастающей нагрузки с клиническим контролем состояния (с постоянным или прерывистым положительным давлением на выдохе). Для этих целей могут быть использованы аппараты типа СИПАП, аппарата Фролова, PARI O-PEP; элементы дыхательной гимнастики А.Н.Стрельниковой, полного дыхания йогов, Цигун-терапии, техники мобилизации грудной клетки и ребер методами мануальной терапии, остеопатии, миофасциального релиза дыхательных мышц; коррекция мышечных триггеров дыхательной мускулатуры. [10,11]. 
Неоспоримым плюсом являются дополнительные аэробные нагрузки, длительность которых должна быть не менее 3-х раз в неделю. Оптимальная продолжительность курса 8-12 недель. У некоторых пациентов, имеющих слабые четырехглавые и ягодичные мышцы возможно применение нервно-мышечной электростимуляции [12].

Физиопроцедуры могут быть применимы при наличии 2-х отрицательных тестов ПЦР /или наличии антител после перенесенной COVID-19 инфекции.

Их целью является обеспечение противовоспалительного (ЭМП СВЧ), противоотечного, репаративно-регенеративного действия (низкочастотная магнитотерапия), противоболевого действия (высокочастотная импульсная магнитотерапия), спазмолитического (синусоидальные модулированные токи), дефиброзирующего (ультразвуковая терапия), улучшение микроциркуляторных процессов (индуктотермия).Пациент должен находится на динамическом наблюдении у врача-терапевта, врача общей практики, лечебной физкультуры, реабилитолога, диетолога, физиотерапевта. В случае необходимости возможна консультация специалиста узкого профиля [13].

Согласно Аналитическому Вестнику № 5, утвержденному Советом Федерации от 11 февраля 2021 года в состав мультидисциплинарной реабилитационной команды должны быть включены следующие специальности: специалист по эргореабилитации, специалист по физической реабилитации, медицинской психологии [14].
Основной проблемой в данный момент времени является отсутствие четких подходов к наблюдению лиц, перенесших короновирусную инфекцию.

Зарубежными исследователями (Klok F.A., 2020) была разработана шкала «The Post-COVID-19 Functional Status (PCFS) Scale», которая позволяет с большой достоверностью оценить функциональное состояние пациентов в пост-ковидном периоде [15].

Ее применение в клинической практике позволит отслеживать динамику общего состояния на различных этапах восстановительного лечения.

Разработка определенных алгоритмов ведения пациентов на амбулаторно-поликлиническом этапе в настоящее время является перспективным направлением, позволяющим значительно снизить нагрузку на первичное звено и оптимизировать подходы к восстановительному лечению.

\section{Выво}

Развитие короновирусной инфекции сопровождается не только тяжелым течением, но и пост-ковидным периодом, длительностью несколько месяцев, значительно ухудшающим общее состояние и снижающим качество жизни больных. Разработка алгоритмов ведения пациентов на данном этапе является залогом успешного их ведения, направленного на восстановление функционального состояния организма.

\section{ЛИТЕРАТУРА}

1. Greenhalgh Trisha, Knight Matthew, $A^{\prime}$ CourtChristine, et al. Management of post-acute covid-19 in primary care. BMJ. 2020; 370: $\mathrm{m} 3026$

2. Малявин А.Г., Адашева Т.В., Бабак С.Л. и др. Медицинская реабилитация больных, перенесших COVID-19 инфекцию. Методические рекомендации. Терапия. 2020; 5 (приложение): 1-48. doi: 10.18565/therapy.2020.5suppl.1-48.

3. Liang Tingbo, Cai Hongliu, Chen Yu, Chen Zuobing, Fang Qiang, Han Weili, Hu Shaohua, Li Jianping, Li Tong, Lu Xiaoyang, Qu Tingting, Shen Yihong, Sheng Jifang, Wang Huafen, Wei Guoqing, Xu Kaijin, Zhao Xuehong, Zhong Zifeng, Zhou Jianying. Ed. Liang Tingbo. Handbook of COVID-19 Prevention and Treatment. 2020.60 p. Available at: http://education.almazovcentre.ru/wpcontent/uploads/2020/03/Spravochnik_po_profilaktike_i_lecheniju_COVID_1 <9.pdf(accessed 11.05.2020).

4. Zhao HM, Xie YX, Wang C. Recommendations for respiratory rehabilitation in adults with COVID-19. Chin Med J (Engl). 2020. [Ahead of print, published online 9 April 2020]. doi:10.1097/CM9.0000000000000848

5. Алашеев А.М., Анисимова Л.Н., Белкин А.А., Вознюк И.А., Иванова Г.Е., Лубнин А.Ю., Лукьянов А.Л., Петриков С.С., Пирадов М.А., Полякова А.В., Сорокоумов В.А., Стаховская Л.В., Суворов А.Ю., Цветков Д.С., Хасанова Д.Р., Шамалов Н.А., Шестопалов Е.Ю., Щеголев А.В. Клинические рекомендации: Вертикализация пациентов в процессе реабилитации. Москва. 2014. 63 С

6. Barazzoni R, Bischoff SC, Breda J, et al. ESPEN expert statements and practical guidance for nutritional management of individuals with SARS-CoV-2 infection 72 Версия 2 (31.07.2020) [published online ahead of print, 2020 Mar 31]. Clin Nutr. 2020; S0261-5614(20)30140-0. doi: 10.1016/j.clnu.2020.03.022

7. Ahmed MZ, Ahmed 0, Aibao Z, Hanbin S, Siyu L, Ahmad A. Epidemic of COVID-19 in China and associated Psychological Problems. Asian J Psychiatr. 2020 Apr 14; 51:102092. doi: 10.1016/j.ajp.2020.102092.

8. Оленская Т.Л., Николаева А.Г., Соболева Л.В. Реабилитация в пульмонологии. Учебно-методическое пособие. Витебск. 2016. С. 90-104.

9. Ларина В.Н., Рыжих А.А., Бикбаева Л.И. Пост-ковидный период: современный взгляд и клинические особенности. Архивъ внутренней медицины. 2021; 11(3): 186-195. D0I: 10.20514/2226-6704-2021-11-3-186-195

10. Ambrosino N, Fracchia C. The role of tele-medicine in patients with respiratory diseases. Expert Rev Respir Med. 2017;11(11):893-900. doi:10.1080/17476348.201 7.1383898 
11. Donner CF, Raskin J, ZuWallack R, et al. Incorporating telemedicine into the integrated care of the COPD patient a summary of an interdisciplinary workshop held in Stresa, Italy, 7-8 September 2017. Respir Med. 2018; 143:91-102. doi: 10.1016/j.rmed.2018.09.003

12. Fossat G, Baudin F, Courtes L, et al. Effect of In-Bed Leg Cycling and Electrical Stimulation of the Quadriceps on Global Muscle Strength in Critically III Adults: A Randomized Clinical Trial. JAMA. 2018; 320 (4):368-378. doi:10.1001/jama.2018.9592

13. Greenhalgh Trisha, Knight Matthew, A'CourtChristine, et al. Management of post-acute covid-19 in primary care. BMJ. 2020; $370: \mathrm{m} 3026$

14. Аналитический вестник № 5 (765). Реабилитация после COVID-19: проблемы и возможности. Материалы заседания Совета по региональному здравоохранению при Совете Федерации Федерального Собрания Российской Федерации (Совет Федерации, 11 февраля 2021 года). Под общей редакцией А.Е. Петрова

15. Klok F.A., Boon G.J. A.M., Barco S., et al. The PostCOVID-19 Functional Status scale: a tool to measure functional status over time after COVID-19. Eur Respir J. 2020; 56(1): 2001494. doi: 10.1183/13993003.01494-2020.

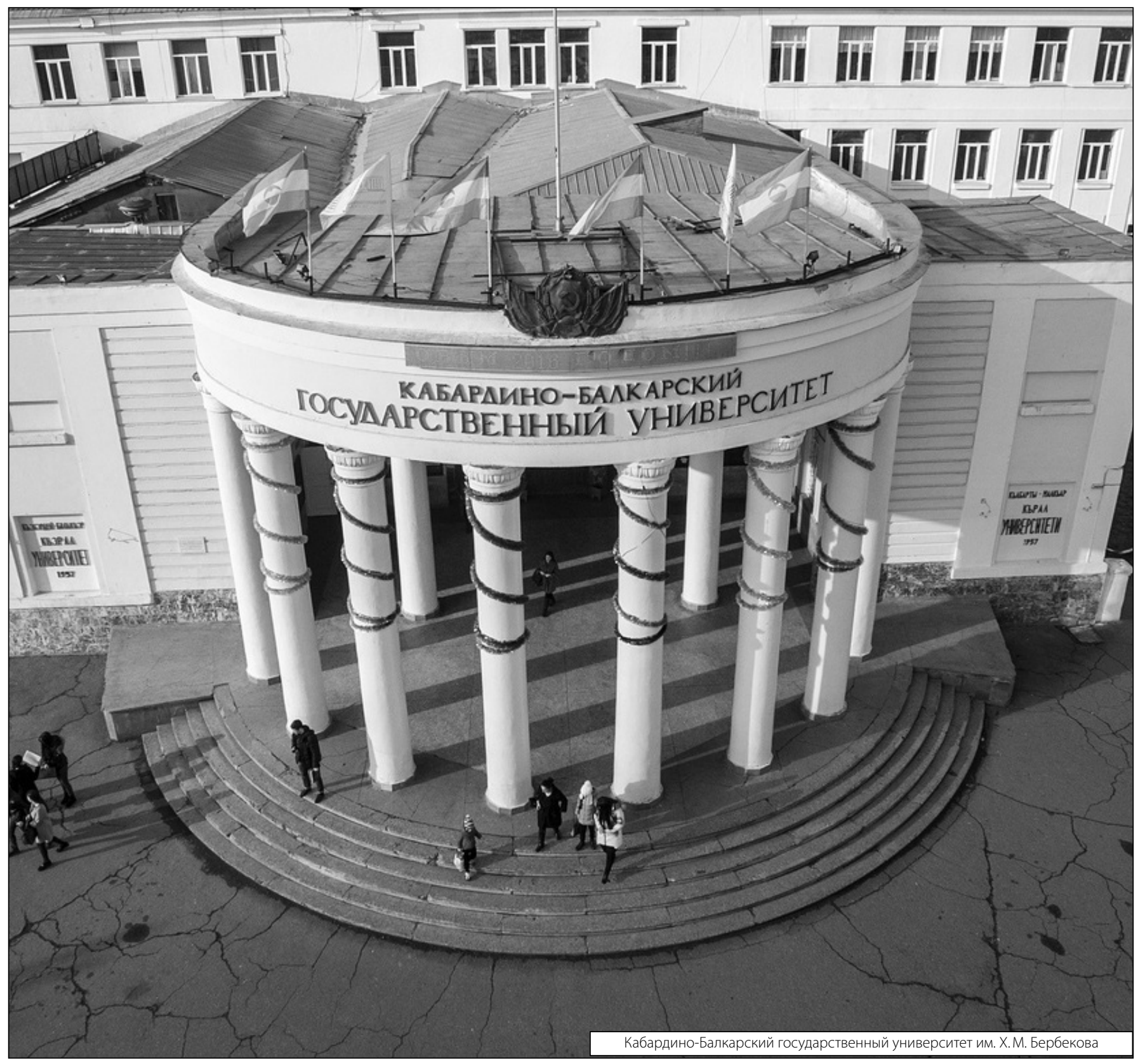

